\title{
Combining deep-inspiration breath hold and intensity-modulated radiotherapy for gastric mucosa-associated lymphoid tissue lymphoma: Dosimetric evaluation using comprehensive plan quality indices
}

Seo Hee Choi ${ }^{1+}$, So Hyun Park ${ }^{2+}$, Jason Joon Bock Lee ${ }^{1}$, Jong Geol Baek ${ }^{1}$, Jin Sung Kim ${ }^{1 *}$ and Hong In Yoon ${ }^{1 *}$ (D)

\begin{abstract}
Background: Although there have been many attempts to increase the therapeutic ratio of radiotherapy for gastric mucosa-associated lymphoid tissue lymphoma (MALToma), only a few planning studies have reported the efficacy of the modern radiotherapy technique till date. Therefore, we performed the dosimetric comparison among 3dimensional conformal radiotherapy (3D-CRT) and intensity-modulated radiotherapy (IMRT) plans, using deepinspiration breath hold $(\mathrm{DIBH})$ or free-breathing $(\mathrm{FB})$ techniques, to determine the most optimal plan for gastric MALTOMa.

Methods: We evaluated 9 patients with gastric MALToma for whom 3D-CRT, step-and-shoot IMRT (sIMRT), volumetric-modulated arc therapy (VMAT), and tomotherapy plans with identical prescribed doses were generated using DIBH or FB computed tomography (CT). Planning target volume (PTV) coverage and non-target doses were calculated for each plan and compared with plan quality metric (PQM) scores.

Results: All 72 plans of 9 patients satisfied our dosimetric goals, and the IMRT plans and 3D-CRT plans had similarly good conformity index values with no differences related to respiratory movement. IMRT plans yielded significantly better doses to the organs-at-risk, and DIBH plans yielded significantly lower liver, heart, and lung $\mathrm{D}_{\text {mean }}$ and spinal cord $D_{\max }$ with smaller irradiated volumes compared to FB plans. For the mean PQM scores, VMAT-DIBH and sIMRT$\mathrm{DIBH}$ yielded the best scores, whereas 3D plans provided reduced beam monitor unit values.

Conclusion: Our findings demonstrate that modern RT technologies (DIBH with VMAT or SIMRT) could potentially provide excellent target coverage for gastric MALToma while reducing doses to organs-at-risk. However, the relevance of the most optimal plan considering clinical outcomes should be confirmed further in a larger patient cohort.
\end{abstract}

Keywords: Mucosa-associated lymphoid tissue lymphoma, Radiotherapy, Planning study, Deep inspiration breath hold, Intensity modulated radiotherapy

\footnotetext{
*Correspondence: JINSUNG@yuhs.ac; YHI0225@yuhs.ac

†'Seo Hee Choi and So Hyun Park contributed equally to this work.

${ }^{1}$ Department of Radiation Oncology, Yonsei Cancer Center, Yonsei University

College of Medicine, 50-1 Yonsei-ro, Seodaemun-gu, Seoul 03722, South

Korea

Full list of author information is available at the end of the article
}

(C) The Author(s). 2019 Open Access This article is distributed under the terms of the Creative Commons Attribution 4.0 International License (http://creativecommons.org/licenses/by/4.0/), which permits unrestricted use, distribution, and reproduction in any medium, provided you give appropriate credit to the original author(s) and the source, provide a link to the Creative Commons license, and indicate if changes were made. The Creative Commons Public Domain Dedication waiver (http://creativecommons.org/publicdomain/zero/1.0/) applies to the data made available in this article, unless otherwise stated. 


\section{Background}

Mucosa-associated lymphoid tissue lymphoma (MALToma) accounts for approximately $19 \%$ of all non-Hodgkin lymphomas and can arise at any extranodal site. In Korea, however, at least half of all MALTomas present as primary gastric lymphomas [1, 2]. Several institutions have reported excellent disease control with radiation therapy alone, supporting the use of modest doses in field radiotherapy (30-40 Gy) for patients with stage I-II gastric MALToma [3-8], although systemic therapy may also be needed in cases involving unsuccessful Helicobacter pylori eradication or $H$. pylor$i$-negative disease, depending on the disease stage.

The recent National Comprehensive Cancer Network $(\mathrm{NCCN})$ guidelines recommend $30 \mathrm{~Gy}$ involved-site radiation therapy for gastric MALToma [9]; this generally involves the entire stomach and adjacent perigastric lymph nodes (if involved). Although this involved-site radiation therapy uses low doses, the doses to the organs-at-risk (OARs) near the stomach, such as the kidneys, small bowel, or liver, must be considered. To date, several planning techniques involving anterior-posterior/ posterior-anterior fields (AP/PA) and 3-dimensional conformal radiotherapy (3D-CRT) have been used to reduce radiation exposure to the OARs to within tolerance limits, and intensity-modulated radiotherapy (IMRT) techniques for gastric MALToma have recently been introduced [10-15]. Despite the dosimetric advantages of these modalities in terms of the doses to the OARs, concerns regarding higher scattered doses have not been resolved [16]. Additionally, the effect of respiratory motion on the accuracy of the treatment has raised concerns, thus limiting the widespread use of IMRT.

Some institutions have implemented the deep-inspiration breath hold (DIBH) technique to address these concerns. However, only a few planning studies have included modern radiotherapy techniques when evaluating the efficacy of this technique [11-13, 17]. Therefore, in the present study, we performed a dosimetric comparison of 3D-CRT and IMRT plans using DIBH or free-breathing (FB) techniques to determine the most optimal treatment plan for gastric MALToma. We additionally compared IMRT plans using various techniques (step-and-shoot IMRT [SIMRT], volumetric-modulated arc therapy [VMAT], and tomotherapy) to identify the most dosimetrically optimal plan, using the plan quality metric (PQM) to ensure an objective assessment.

\section{Materials and methods Patient selection}

Among 20 patients who received definitive radiotherapy for gastric MALToma at our institution between 2016 and 2017, we selected only patients who underwent computed tomography $(\mathrm{CT})$ simulation scans using both
FB and DIBH, to make 4 different plans (3D CRT, sIMRT, VMAT, and tomotherapy) per CT scan type (FB or DIBH) of each patient. A total of 9 patients who received definitive radiotherapy for localized gastric MALToma were selected consecutively for this planning study. All cases were either $H$. pylori-positive but unresponsive to $H$. pylori irradiation or $H$. pylori-negative. Before the start of radiotherapy, each patient underwent CT simulation scans (Aquilion LB; Toshiba Medical System, Tokyo, Japan) using both FB and DIBH, per our institutional protocol after at least $4 \mathrm{~h}$ of fasting. The patients received intravenous contrast agents and were immobilized in a supine position with both arms raised above the head. The range for CT scan was determined to include all the OARs (such as the lungs, heart, kidneys, and bowel) that should be considered in planning. In our institution, patients were scanned from approximately the level of the 7th thoracic vertebral body inferiorly to the level of the 4th lumbar vertebral body. Care was taken to include the base of the heart and both kidneys. DIBH CT scans were performed while the patient held his/her breath using the abdomen and chest motion self-control (Abches) system, as described in our previous report [18].

In all patients, the gross tumor volume (GTV) was defined as the whole stomach. However, different clinical target volumes (CTVs) were set for FB and DIBH CT, as the former must incorporate the concept of an internal target volume (ITV) to account for respiratory movement during treatment. The CTV was defined as the GTV plus a $1.5-\mathrm{cm}$ margin for FB CT, and GTV plus a $1.0-\mathrm{cm}$ margin for DIBH CT. To account for set-up errors, the planning target volume (PTV) was defined as the CTV plus a $0.5-\mathrm{cm}$ margin for all CT scans. For all plans, the prescribed dose was equal to $30 \mathrm{~Gy}$ in 20 fractions.

Four different plans, including one 3D-CRT plan and three IMRT plans (sIMRT, VMAT, and tomotherapy), were generated per CT scan type (FB or $\mathrm{DIBH}$ ) to yield 8 plans per case. Therefore, we compared 72 plans for 9 patients based on the planning modality and respiration control methods. This study was approved by the institutional review board (IRB) of the Yonsei University Health System (4-2017-1035).

\section{Planning techniques}

The FB and DIBH CT images and all datasets were transferred to treatment planning systems. The 3D-CRT, sIMRT, and VMAT plans were created with the RayStation (RayStation 5.0; RaySearch Laboratories, Stockholm, Sweden), and tomotherapy plans were generated with a TomoTherapy Hi-Art System (Accuray Inc., Madison, WI, USA). The 3D-CRT plans comprised four 10-MV energy beams, arranged as anterior-posterior opposed 
beams and two lateral beams. The ${ }_{s}$ IMRT plans were created using 7 angles $\left(0^{\circ}, 50^{\circ}, 100^{\circ}, 150^{\circ}, 210^{\circ}, 260^{\circ}\right.$, and $315^{\circ}$ ) and a collimator angle of $90^{\circ}$, according to clinical experience. The VMAT plans used 2 full 6-MV arcs. The tomotherapy plans comprised helical beams optimized using a field width of $1.05^{\circ}$, modulation factor of 2.4 , and pitch of 0.3. Each plan aimed to ensure 95\% coverage of the PTV to the prescribed dose, with critical organ dose limits of 12.5 Gy and 10.0 Gy for the mean doses $\left(D_{\text {mean }}\right)$ to the liver and kidneys, respectively.

\section{Dosimetric parameters for plan evaluation}

A radiation oncologist following the Radiation Therapy Oncology Group (RTOG) contouring atlases defined each OAR contour. Each OAR contour was contoured, and the body contour was automatically segmented using the "Whole Body" contouring tool with the MIM software (Cleveland, Ohio) in all patients. The dose distributions for each plan were analyzed using dose-volume histograms and dose distributions, and doses to the OARs were evaluated using the following criteria:

1) $D_{\text {mean }}$ to each kidney, liver, and heart

2) Maximum dose $\left(D_{\max }\right)$ to the bowel and spinal cord

3) $D_{\text {mean }}$ to both lungs, volume of both lungs receiving $\geq 5 \mathrm{~Gy}\left(\mathrm{~V}_{5}\right)$, and volume of both lungs receiving $\geq 20$ Gy $\left(V_{20}\right)$

The PTV doses were evaluated using the following parameters to evaluate target coverage and homogeneity:

1) Percent volume of the PTV receiving at least $95 \%$ of the prescription dose $\left(\mathrm{TV}_{95}\right)$

2) Homogeneity index (HI): $\mathrm{HI}=\mathrm{D}_{5} / \mathrm{D}_{95}$

Where $\mathrm{D}_{5}$ and $\mathrm{D}_{95}$ represent the minimum doses to 5 and $95 \%$ of the PTV, respectively [19].

3) Conformity index $(\mathrm{CI}): \mathrm{CI}=\mathrm{BV}_{95} / \mathrm{PTV}$

Table 1 Quality scores for each objective

\begin{tabular}{|c|c|c|c|c|c|c|c|c|c|c|c|}
\hline \multirow[b]{2}{*}{ Score } & \multirow{2}{*}{\multicolumn{3}{|c|}{$\begin{array}{l}\text { Target } \\
\text { PTV }\end{array}$}} & \multicolumn{8}{|c|}{ Organ-at-risk (OAR) } \\
\hline & & & & Rt. kidney & Lt. kidney & Spinal cord & Liver & Heart & Lung & & Bowel $^{a}$ \\
\hline & $\mathrm{D}_{95}(\mathrm{cGy})$ & $\mathrm{HI}$ & $\mathrm{Cl}$ & $D_{\text {mean }}(c G y)$ & $D_{\text {mean }}(c G y)$ & $\mathrm{D}_{\max }(\mathrm{cGy})$ & $\mathrm{D}_{\text {mean }}(\mathrm{cGy})$ & $D_{\text {mean }}(c G y)$ & $D_{\text {mean }}(c G y)$ & V20 $\left(\mathrm{cm}^{3}\right)$ & $D_{\max }(c G y)$ \\
\hline 0 & $<2550$ & 1.0 & - & $>1800$ & $>1800$ & $>3000$ & $\geq 2000$ & $>1000$ & $\geq 900$ & $>2000$ & \\
\hline 1 & 2550 & 0.9 & $<0.2,>1.8$ & 1800 & 1800 & 3000 & 1950 & 1000 & 950 & 2000 & \\
\hline 2 & 2600 & 0.8 & $0.2,1.8$ & 1700 & 1700 & 2900 & 1900 & 950 & 900 & 1900 & \\
\hline 3 & 2650 & 0.7 & $0.3,1.7$ & 1600 & 1600 & 2800 & 1850 & 900 & 850 & 1800 & \\
\hline 4 & 2700 & 0.6 & $0.4,1.6$ & 1500 & 1500 & 2700 & 1800 & 850 & 800 & 1700 & \\
\hline 5 & 2750 & 0.5 & $0.5,1.5$ & 1400 & 1400 & 2600 & 1750 & 800 & 750 & 1600 & \\
\hline 6 & 2800 & 0.4 & $0.6,1.4$ & 1300 & 1300 & 2500 & 1700 & 750 & 700 & 1500 & 2700 \\
\hline 7 & 2850 & 0.3 & $0.7,1.3$ & 1200 & 1200 & 2400 & 1650 & 700 & 650 & 1400 & 2750 \\
\hline 8 & 2900 & 0.2 & $0.8,1.2$ & 1100 & 1100 & 2300 & 1600 & 650 & 600 & 1300 & 2800 \\
\hline \multirow[t]{2}{*}{9} & 2950 & 0.1 & $0.9,1.1$ & 1000 & 1000 & 2200 & 1550 & 600 & 550 & 1200 & 2850-2950 \\
\hline & & & & & & & & & & & $3050-3150$ \\
\hline 10 & 3000 & 0.0 & 1.0 & 950 & 950 & 2100 & 1500 & 550 & 500 & 1100 & 3000 \\
\hline 11 & & & & 900 & 900 & 2000 & 1450 & 500 & 450 & 1000 & \\
\hline 12 & & & & 850 & 850 & 1900 & 1400 & 450 & 400 & 900 & \\
\hline 13 & & & & 800 & 800 & 1800 & 1350 & 400 & 350 & 800 & \\
\hline 14 & & & & 750 & 750 & 1700 & 1300 & 350 & 300 & 700 & \\
\hline 15 & & & & 700 & 700 & 1600 & 1250 & 300 & 250 & 600 & \\
\hline 16 & & & & 650 & 650 & 1500 & 1200 & 250 & 200 & 500 & \\
\hline 17 & & & & 600 & 600 & 1400 & 1150 & 200 & 150 & 400 & \\
\hline 18 & & & & 550 & 550 & 1300 & 1100 & 150 & 100 & 300 & \\
\hline 19 & & & & 500 & 500 & 1200 & 1050 & 100 & & 200 & \\
\hline 20 & & & & 400 & 400 & 1100 & 1000 & 50 & & 100 & \\
\hline
\end{tabular}

The sum of scores for each objective is defined as the "raw plan quality metric (PQM)". The maximum score was set to 178 , and the PQM (\%) was determined as the percent of the Raw PQM to Max PQM for each plan

a Bowel scoring was conducted differently because part of the bowel is included in the PTV, and therefore, the bowel dose partly reflects the PTV coverage Abbreviations: PTV planning target volume, $\mathrm{HI}$ homogeneity index, $\mathrm{Cl}$ conformity index 
Where $\mathrm{BV}_{95}$ represents the volume of the body receiving $95 \%$ of the prescribed dose [20].

In our practice, we used a radiotherapy plan analysis program (Plan $\mathrm{IQ}^{\mathrm{rm}}$, Sun Nuclear co, Melbourne, FL, USA) to generate scores for these dosimetric goals according to the PQM. The scores were based on the constraints and dose-volume histograms of the planning results and assigned to each evaluation object according to the calculated dose [21, 22]. The quality scores for each objective are shown in Table 1. The PQM (\%) represents the ratio of the raw $\mathrm{PQM}$ to the maximum $\mathrm{PQM}$. The raw $\mathrm{PQM}$ is the evaluation score of each treatment plan in the score template. The maximum PQM is the highest score that the treatment plan can achieve and is the sum of the highest scores for each objective set by the user of plan IQ (set to 178). By using plan IQ, it is possible to create the score template for each objective that users want to evaluate. Depending on the treatment sites and prescribed dose, it is rated at a higher score compared to other scores for significant objectives. Because higher scores can be assigned to important OARs, dosimetric weighting is determined by clinical importance. To explain the score template, the score template for the kidney and lung $\mathrm{D}_{\text {mean }}(\mathrm{Gy})$ is shown in Additional file 1: Figure S1.

\section{Statistical analysis}

The Wilcoxon signed-rank test and Friedman test were used for the group-wise statistical comparison of the 8 planning techniques (3D-DIBH, 3D-FB, sIMRT-DIBH, sIMRT-FB, VMAT-DIBH, VMAT-FB, Tomo-DIBH, and Tomo-FB). A $p$-value of $<0.05$ was considered statistically significant. Statistical analyses were performed using SPSS software (Ver. 23.0; SPSS Inc., Chicago, IL, USA).

\section{Results}

Target coverage, conformity, and homogeneity

All plans satisfied the criteria for good PTV coverage, conformity, and homogeneity. Accordingly, the group-wise comparison of PTV dose distribution involved the $\mathrm{TV}_{95}$ and $\mathrm{D}_{95}$ values of the 8 techniques. Although all $D_{95}$ values were larger than $95 \%$ of the prescribed dose, the tomotherapy plans yielded the best result, followed by sIMRT, VMAT, and 3D-plans, with no differences between the DIBH and FB plans. Statistically, the IMRT plans were significantly better than 3D-CRT plans, sIMRT plans were significantly better than VMAT plans, and tomotherapy plans were significantly better than ${ }_{S}$ IMRT plans. The mean $\mathrm{D}_{95}$ values of the 3D-DIBH, 3D-FB, sIMRT-DIBH, sIMRT-FB, VMAT-DIBH, VMAT-FB, Tomo-DIBH, and Tomo-FB plans are shown in Table 2 and Fig. 1(a) $(p<0.001)$. The detailed results of statistical analyses are presented in Additional file 2: Supplementary text 1.

Regarding the $\mathrm{TV}_{95}$, significantly larger irradiated volumes were observed with FB plans than with $\mathrm{DIBH}$ plans (mean: $1721 \mathrm{cc}$ for $\mathrm{FB}>1372 \mathrm{cc}$ for $\mathrm{DIBH}, p<$ 0.001 ), indicating the need to compensate for respiratory movement. The mean $\mathrm{TV}_{95}$ values of the $3 \mathrm{D}-\mathrm{DIBH}$, 3D-FB, sIMRT-DIBH, sIMRT-FB, VMAT-DIBH, VMAT-FB, Tomo-DIBH, and Tomo-FB plans are shown in Table 2 and Fig. 1(b) $(\mathrm{p}<0.001)$. On statistical analyses, 3D plans yielded significantly higher $\mathrm{TV}_{95}$ values than IMRT plans, whereas VMAT plans had the lowest $\mathrm{TV}_{95}$ when plans using the same respiration modality were compared. The results of statistical analyses are presented in Additional file 2: Supplementary text 2.

Regarding homogeneity, the 3D, sIMRT, and VMAT plans yielded similar $\mathrm{HI}$ values, regardless of respiratory movement. Although plans showed favorable homogeneity, the tomotherapy plans were the most superior, regardless of respiratory movement. The mean $\mathrm{HI}$ values of the 3D-DIBH, 3D-FB, sIMRT-DIBH, sIMRT-FB, VMAT-DIBH, VMAT-FB, Tomo-DIBH, and Tomo-FB plans are shown in Table 2 and Fig. $1(\mathrm{c})(p<0.001)$. The detailed results of statistical analyses are presented in Additional file 2: Supplementary text 3.

Regarding dose conformity, the 3D plans yielded the worst CI values, followed by IMRT plans (which had similar CI values). Among the IMRT plans, sIMRT and VMAT yielded the best CI values. As shown in Fig. 2, which demonstrates the isodose lines, 3D-DIBH and $3 \mathrm{D}-\mathrm{FB}$ were the least conformal plans, followed by Tomo-DIBH and Tomo-FB. No differences were observed according to respiratory movement. The mean $\mathrm{CI}$ values of the 3D-DIBH, 3D-FB, sIMRT-DIBH, sIMRT-FB, VMAT-DIBH, VMAT-FB, Tomo-DIBH, and Tomo-FB plans are shown in Table 2 and Fig. $1(\mathrm{~d})(p<$ $0.001)$. The detailed results of statistical analyses are presented in Additional file 2: Supplementary text 4, and differences in homogeneity and conformity among the plans are demonstrated in an example of the plans with isodose lines (Fig. 2).

\section{OAR doses}

The DIBH plans, which involved smaller irradiated volumes, yielded lower kidney $D_{\text {mean }}$ values on both sides relative to the FB plans. The $D_{\text {mean }}$ values were the highest for the 3D-CRT plans, followed by tomotherapy, sIMRT, and VMAT, although these differences were not significant. Similarly, the DIBH plans yielded significantly lower $D_{\text {mean }}$ values for the liver, heart, and lungs, compared to FB plans. For these OARs, the VMAT-DIBH and sIMRT-DIBH plans were significantly superior to the others, 


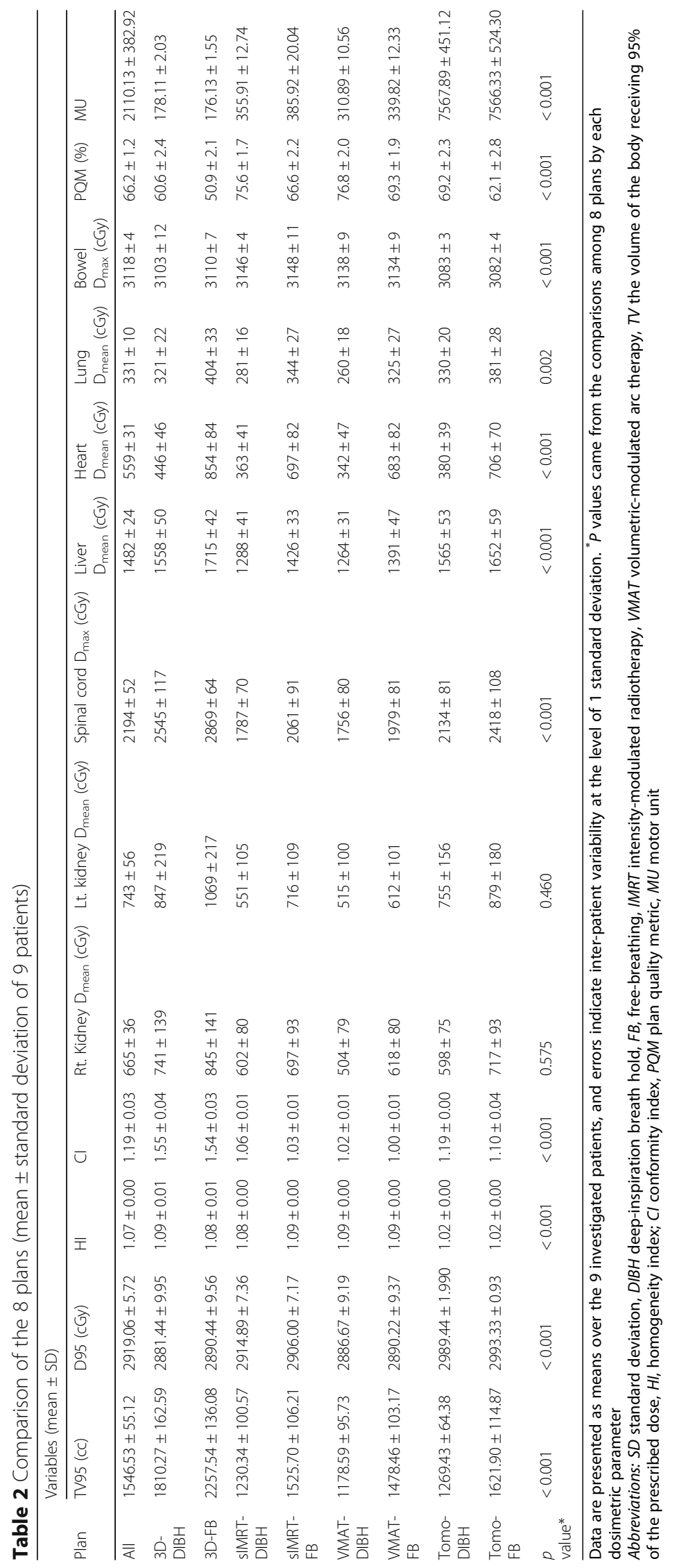



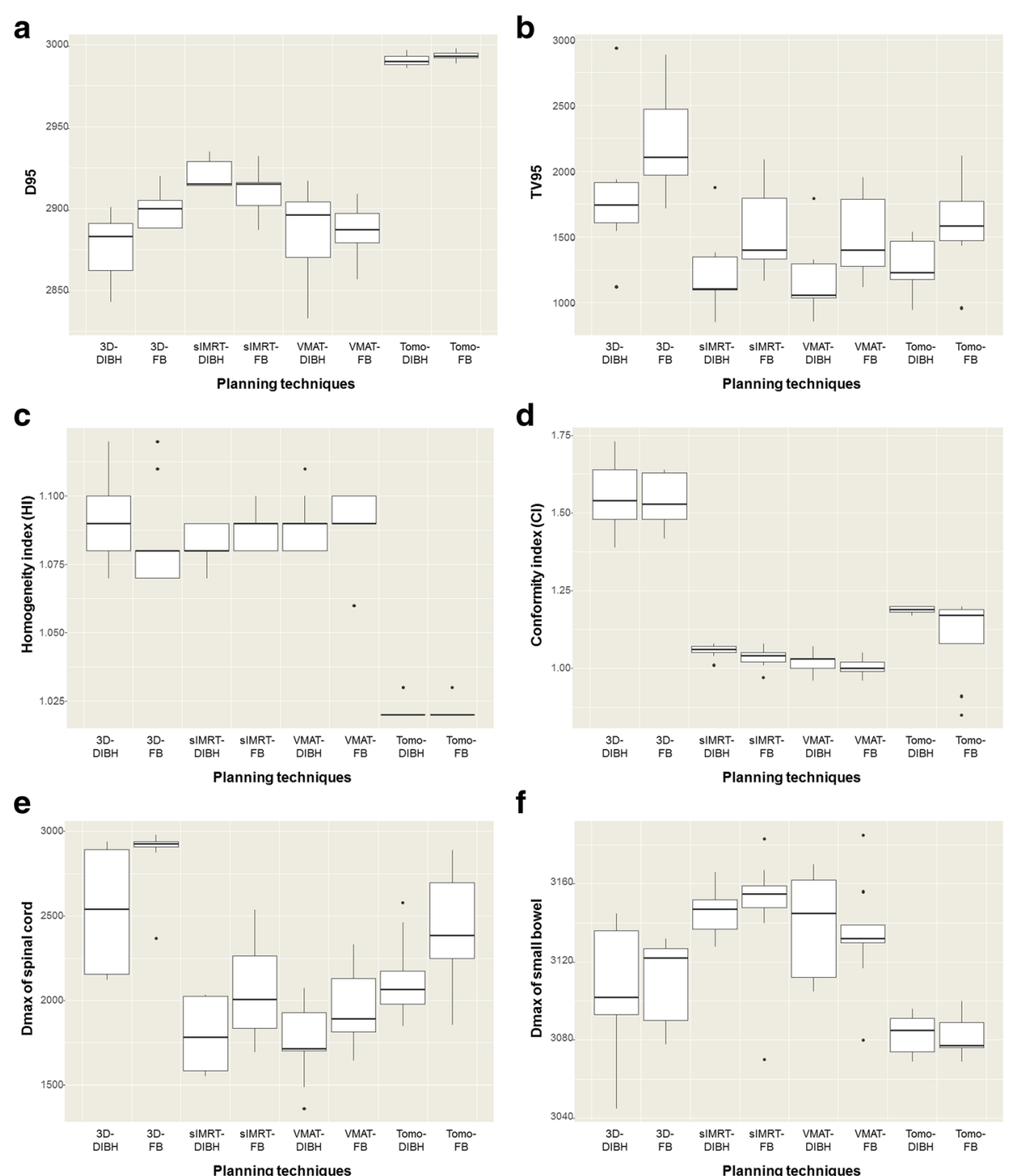

Fig. 1 Boxplots of (a) the doses to 95\% of the PTV $\left(D_{95}\right)$, (b) percent volume of the PTV receiving at least 95\% of the prescription dose (TV $V_{95}$ ), (c) homogeneity index $(\mathrm{HI})$, (d) conformity index $(\mathrm{Cl})$, (e) $\mathrm{D}_{\max }$ of the spinal cord, and $(\mathbf{f}) \mathrm{D}_{\max }$ of the small bowel of the 8 different plan groups. ${ }^{*}$ The significant differences between groups are shown in the Supplementary text

whereas the Tomo-FB and 3D-FB plans yielded the worst outcomes. As shown in Fig. 2, the DIBH plans yielded smaller irradiated volumes for the liver and kidneys, compared to the corresponding FB plans.

For the spinal cord, DIBH plans yielded significantly lower $\mathrm{D}_{\max }$ values, compared to FB plans. 3D-CRT plans yielded the highest values, followed by tomotherapy, ${ }_{\text {sIMRT }}$ and VMAT plans. The values and results of statistical analyses are presented in Fig. 1(e) and Additional file 2: Supplementary text 5 . In contrast, the $D_{\max }$ values for the small bowel did not differ significantly among the plans (Fig. 1(f)), although the ${ }_{s}$ IMRT plans and VMAT plans yielded the highest values, followed by the 3D plans and Tomo plans. Respiratory movement also had no effect on the small bowel $D_{\max }$ values. The results of statistical analyses are presented in Additional file 2: Supplementary text 6, and the mean values for each OAR with each plan are shown in Table 2.

\section{PQM}

As shown in Table 1, the PQM score (\%) of each plan was determined after weighting each dosimetric factor according to clinical importance. The VMAT-DIBH plan and ${ }_{S}$ IMRT-DIBH plan acquired the best mean PQM scores (\%) of 76.8 and 75.6, respectively. The VMAT-FB, Tomo-DIBH, sIMRT-FB, and Tomo-FB plans acquired mean PQM scores of 69.3, 69.2, 66.6, and 62.1, respectively. Both 3D-DIBH and FB plans acquired the lowest PQM scores (\%) of 60.6 and 50.9, respectively (Table 2). All DIBH plans yielded significantly superior PQM 


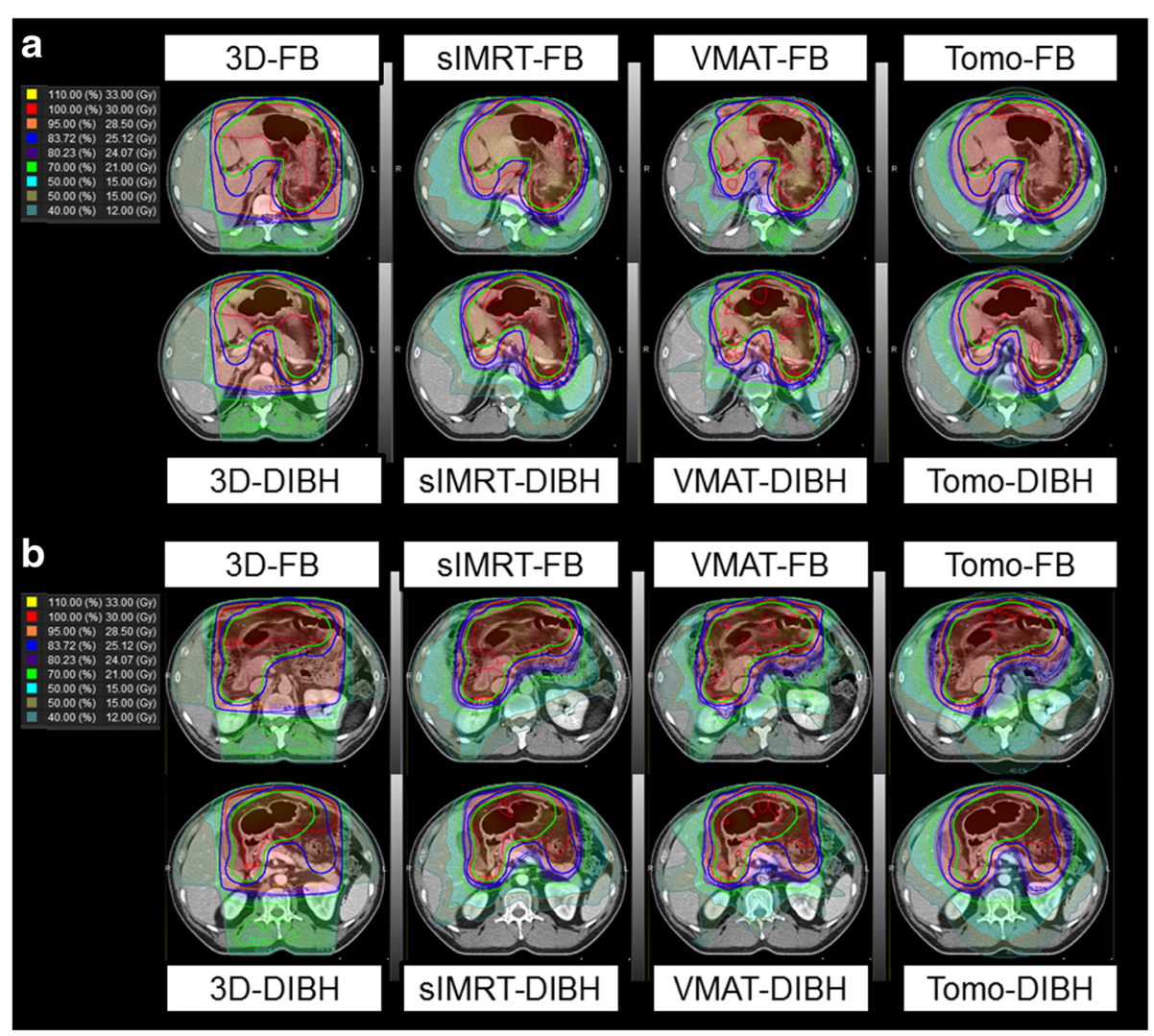

Fig. 2 Treatment plans for 1 patient; (a) isodose lines in the image showing the curvature of the stomach, (b) isodose lines in the image showing the irradiation dose to both the kidneys. In Fig. 2(a), the difference in $\mathrm{D}_{95}, \mathrm{TV}_{95}, \mathrm{HI}$, and $\mathrm{Cl}$ values among treatment plans can be compared visually to some extent. In Fig. 2(b), the difference between the right and left kidney $D_{\text {mean }}$ liver $D_{\text {mean, }}$ and bowel $D_{\max }$ doses among treatment plans can be compared visually to some extent
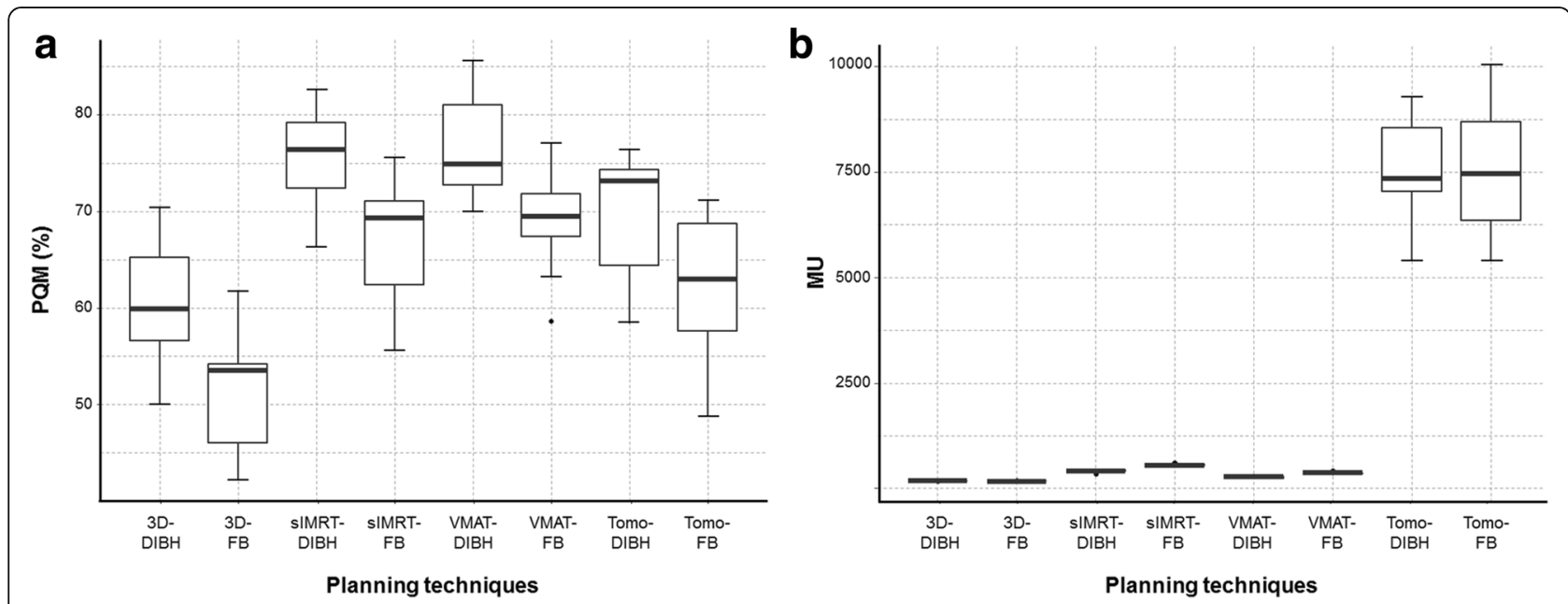

Fig. 3 Boxplots of (a) plan quality metric (PQM) scores and (b) motor units (MU) of the 8 different plan groups. DIBH, deep-inspiration breath hold; $F B$, freebreathing; 3D, 3-dimensional conformal radiotherapy; IMRT, intensity-modulated radiotherapy; VMAT, volumetric-modulated arc therapy; Tomo, tomotherapy 
Table 3 The ranking of each dosimetric variable (mean value) and scores generated by summing all rankings of the 8 plans

\begin{tabular}{|c|c|c|c|c|c|c|c|c|}
\hline Variables & 3D-DIBH & $3 \mathrm{D}-\mathrm{FB}$ & sIMRT-DIBH & sIMRT-FB & VMAT-DIBH & VMAT-FB & Tomo-DIBH & Tomo-FB \\
\hline $\mathrm{TV}_{95}$ & 7 & 8 & 3 & 5 & 1 & 4 & 2 & 6 \\
\hline$D_{95}$ & 7 & 4 & 3 & 4 & 6 & 5 & 2 & 1 \\
\hline $\mathrm{HI}$ & 7 & 4 & 3 & 5 & 6 & 8 & 1 & 2 \\
\hline $\mathrm{Cl}$ & 8 & 7 & 4 & 3 & 2 & 1 & 6 & 5 \\
\hline Kidney $D_{\text {mean }}$ & 7 & 8 & 3 & 5 & 1 & 4 & 2 & 6 \\
\hline Spinal cord $D_{\max }$ & 8 & 6 & 2 & 4 & 1 & 3 & 5 & 7 \\
\hline Liver $D_{\text {mean }}$ & 5 & 8 & 2 & 4 & 1 & 3 & 6 & 7 \\
\hline Heart $D_{\text {mean }}$ & 4 & 8 & 2 & 6 & 1 & 5 & 3 & 7 \\
\hline Lung $D_{\text {mean }}$ & 3 & 8 & 2 & 6 & 1 & 4 & 5 & 7 \\
\hline Bowel $D_{\max }$ & 3 & 4 & 7 & 8 & 6 & 5 & 1 & 2 \\
\hline $\mathrm{MU}$ & 2 & 1 & 5 & 6 & 3 & 4 & 8 & 7 \\
\hline Sum & 61 & 66 & 36 & 56 & 29 & 46 & 41 & 57 \\
\hline
\end{tabular}

Abbreviations: DIBH deep-inspiration breath hold, FB free-breathing, IMRT intensity-modulated radiotherapy, VMAT volumetric-modulated arc therapy, TV the volume of the body receiving $95 \%$ of the prescribed dose, $\mathrm{HI}$ homogeneity index, $\mathrm{Cl}$ conformity index, $\mathrm{MU}$ motor unit

scores to the corresponding FB plans (Fig. 3(a)). Although VMAT-DIBH and SIMRT-DIBH were significantly better than most other plans, these two plans were not significantly different $(p=0.066)$. The VMAT-FB and ${ }_{S}$ IMRT-FB plans were significantly better than 3D-CRT plans (DIBH or FB), but significantly worse than those obtained using the DIBH technique (VMAT-DIBH or ${ }_{s}$ IMRT-DIBH). When we further scored the plans by summing all items at a 1:1 ratio (not weighting each dosimetric factor according to clinical importance), the same plan quality rankings as PQM (weighting each dosimetric factor by users according to clinical importance) were achieved. As shown in Table 3,

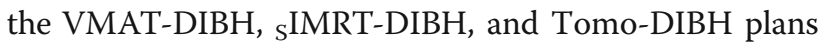
yielded the best results, and all were superior to the corresponding $\mathrm{FB}$ plans. The $3 \mathrm{D}-\mathrm{DIBH}$ and $3 \mathrm{D}-\mathrm{FB}$ plans yielded the most inferior scores for almost all factors.

\section{Motor unit and clinical meaning}

The IMRT plans yielded significantly higher monitor unit (MU) values, whereas the 3D-CRT plans yielded significantly lower MU values relative to the other modalities. Although the VMAT and ${ }_{S}$ IMRT plans did not yield significantly different MU values, all were significantly lower than those of tomotherapy plans. The Tomo-DIBH and Tomo-FB plans required substantially high mean MU values (7567 and 7566, respectively; Table 2), and the MU values did not significantly differ between the DIBH and corresponding FB plans (Fig. 3(b)).

We note that when performing actual treatments using the DIBH technique, beam irradiation can be performed only when the patient is holding his/her breath (intervals of $\sim 15$ to $20 \mathrm{~s}$ ). As free breathing must be allowed between the periods of breath holding, the total treatment time in an actual clinic setting may be longer than expected. In addition, patient training time, DIBH device set-up time, and accurate monitoring in the treatment room are required. Therefore, the time required for actual treatment could be significantly increased if the IMRT and DIBH techniques are combined.

\section{Discussion}

In this study, modern radiotherapy plans combining IMRT and DIBH (VMAT-DIBH, sIMRT-DIBH) were found to be significantly superior to $3 \mathrm{D}$ plans for gastric MALToma. We further observed that tomotherapy plans had exceptionally high MU values and yielded no notable benefits in this context. Although it remains unclear whether DIBH or IMRT is more useful in a clinical setting, VMAT-FB seems to yield better dosimetric outcomes when compared to 3D-DIBH. One of the strengths of this study is that we performed a dosimetric comparison among various modern radiotherapy techniques combining DIBH and IMRT and suggested the most appropriate treatment combination strategy regarding target coverage, OAR doses, and comprehensive evaluation for gastric MALToma.

Since the Memorial Sloan-Kettering Cancer Center (MSKCC) first described the treatment of gastric MALToma with RT alone [3], subsequent studies have shown favorable results in various populations, including patients with $H$. pylori-independent disease [4-8]. However, significant variations in stomach size and shape, digestive movement, and respiratory motion are known to cause uncertainty during the simulation and delivery of treatment to the stomach, and a safe, efficient irradiation technique that can optimally overcome these uncertainties with adequate margins has not been well established. The simplest approach to this issue involves 
the addition of an adequate margin to the CTV. Previous studies of RT planning techniques for gastric lymphoma have recommended a PTV comprising the CTV plus a $1.5-2-\mathrm{cm}$ margin in all directions $[10,12]$ for the delivery of radiation in a FB state. Other studies have used respiratory synchronized 4D-CT images to provide information about respiratory-induced organ motion during treatment planning and minimize motion uncertainties [13, 23]. Specifically, Matoma et al. [17] compared the usefulness of $4 \mathrm{D}-\mathrm{CT}$ vs. a uniform margin for the treatment planning of gastric MALToma, and found that the former yielded a significantly smaller mean PTV volume, with significantly lower mean doses to the liver and heart. According to the International Lymphoma Radiation Oncology Group (ILROG) guidelines for the CTV [24], the entire stomach should be considered to harbor disease even if the tumor appears confined to one area, and abnormal or suggestive perigastric lymph nodes can be included in the CTV. Respiratory motion-induced changes in stomach position should be detected using 4D-CT simulation or fluoroscopy when determining the ITV, and an additional margin of approximately $1 \mathrm{~cm}$ is often added to the CTV for this purpose. The PTV (normally $\sim 1 \mathrm{~cm}$ ) should account for setup variations. Moreover, radiotherapy planning based on $4 \mathrm{D}$-positron-emission tomography (PET)-CT/4D-CT together with online cone-beam CT might be helpful to define PTV margins used for optimizing individual target coverage and estimating interfractional or intrafractional gastric movement [25].

DIBH has become a standard cardiac-sparing technique during the treatment of left-sided breast cancer [26-29], and several experts in lymphoma treatment centers have recently reported the use of DIBH for mediastinal lymphoma [30-33]. In most patients, DIBH reduces heart and lung doses by elongating the heart, resulting in greater separation from the target volume and increasing the lung volume. In DIBH, smaller PTV margins could be applied to further accentuate the organ-sparing benefit. Therefore, this respiratory technique is being considered for use during the treatment of other organs (i.e., liver and stomach) at various specialized institutions, including ours. More recently, Wang et al. demonstrated that there could exist substantial interfractional variation in stomach volume despite treatment with breath-hold and restriction of oral intake, and daily CT image guidance RT (CT-IGRT), in combination with a DIBH, enabled better target coverage with even smaller PTV margins $(0.5-1.0 \mathrm{~cm})$ while treating gastric MALToma [34].

Several investigators have also described the use of IMRT for the treatment of mediastinal lymphoma [32, $35,36]$. As expected, IMRT improves target conformity and reduces OAR doses. However, this technique also increases the volume of low-dose exposure in tissues such as the lungs, heart, and breasts. Notably, the use of the IMRT or DIBH technique in the treatment of Hodgkin lymphoma results in better protection of the heart and lungs [30,31,37]. Therefore, the combination of IMRT and DIBH would be expected to further reduce the doses to the OARs. In addition to the information on the breath-hold technique included in the ILROG guidelines [38], more number of clinical attempts is ongoing to combine IMRT and DIBH effectively for Hodgkin lymphoma.

In contrast to the situation with Hodgkin lymphoma, only a few studies have evaluated the use of modern radiotherapy techniques for gastric MALToma. In the first planning study for gastric MALToma, conducted by the MSKCC to determine the most advantageous technique [10], the PTV was defined as the CTV plus a $2-\mathrm{cm}$ margin to account for respiratory-induced movement of the stomach during FB. In a comparison of AP/ PA, 3D-CRT, and IMRT plans, 4-field 3D-CRT markedly decreased the dose to the kidneys when this organ overlapped with the PTV, and the findings with IMRT plans suggested that the kidney and liver doses could be incrementally improved in selected patients. Two relevant studies were also reported by Korean researchers. Lim et al. [12] retrospectively compared 2 different planning techniques (2D and 3D-CRT) for gastric MALToma based on CT with a FB status; the PTV was defined as the CTV plus a $1-1.5-\mathrm{cm}$ margin, and an additional $1-\mathrm{cm}$ margin was added in the craniocaudal direction to compensate for respiratory-induced stomach motion. For 3D-CRT plans, AP/PA or 3-4 non-coplanar fields were used according to the physician's preference. Although that retrospective study compared treatment outcomes rather than dosimetric planning, the 3D-CRT plans yielded significantly better PTV coverage, conformity, and kidney doses on both sides when compared to 2D-RT plans, without compromising the oncologic outcomes. Furthermore, Bae at al. [13] compared 5 planning techniques (AP/PA, 4-field, 3D-CRT, IMRT with only coplanar beams, and IMRT with a few non-coplanar beams). 4D-CT was conducted under a FB status, ITV was defined as the sum total of the entire stomach at every respiratory stage, and CTV was defined as the ITV plus a $1-\mathrm{cm}$ margin. The authors observed the highest mean kidney and liver doses with the AP/PA plan and a 4-field plan, respectively. As observed in our study, Bae and colleagues observed better conformity and hepatic toxicity with IMRT plans, but found no significant difference between the coplanar and non-coplanar IMRT plans. Unlike our study, however, these previous studies were limited by a lack of comparison with the DIBH technique and with more recent IMRT plans (e.g., VMAT and tomotherapy). In our 
institution, we defined CTVs as the GTV plus a $1.5-\mathrm{cm}$ margin for FB plans, and GTV plus a 1.0- $\mathrm{cm}$ margin for DIBH plans, without 4D-CT scans. There was only a small difference in CTV margins compared to that in other planning studies. Moreover, considering that this study was a dosimetric study for comparing optimal radiotherapy planning techniques in patients, the conclusion would not change and this seems reasonable compared to other studies.

Similar planning studies have been conducted abroad, although neither modern DIBH nor various IMRT planning techniques have been evaluated. A similar planning study that compared four-field 3D-CRT, half-field RT, and IMRT was performed by Japanese researchers [11]. In that study, planning CT was performed during shallow exhale and inhale phases; the CTVs from both phases were fused, peristalsis margins were added to obtain the ITV, and the PTV was generated by expanding the ITV by $1 \mathrm{~cm}$ in all directions. The IMRT and half-beam methods were found to reduce the doses to the kidneys and liver, compared to 3D-CRT. In a retrospective Chinese study [23], the dosimetric superiority and efficacy, toxicity, and quality of life were investigated in patients with gastric diffuse large B-cell lymphoma who received IMRT. IMRT was performed with a FB status, and the PTV was defined as the CTV plus 1-2 $\mathrm{cm}$ in all directions. The 5-year overall survival, progression-free survival, and locoregional control rates were 80,75 , and 93\%, respectively, with excellent target coverage and long-term global and functional quality of life scores.

The application of DIBH to gastric lymphoma might differ significantly with respect to the dose and target location. The use of DIBH requires 10-20 min of coaching during the treatment simulation and prior to administration of the first fraction. A patient is expected to hold their breath for $10-20 \mathrm{~s}$ per respiratory cycle, during which radiation must be delivered. This limitation prolongs the daily treatment time by a few minutes, and the addition of IMRT can further affect the treatment time. Additionally, each treatment requires a very sophisticated protocol. However, patients with gastric lymphoma account for only a small proportion of departmental workloads, and therefore, these limitations may be outweighed by the improved treatment accuracy and reduction in late adverse events.

This study had a few limitations. First, the relatively small sample size might have limited our ability to make firm recommendations regarding the usefulness of DIBH. Second, as this was a treatment planning study, we could not demonstrate the oncologic outcomes or prevalence of secondary malignancies after long-term follow-up in the same cohort. Still, we note that almost 2 years have passed since our institution actually used this technique, and no treatment failures have been reported, although a long follow-up duration would be needed given the late disease recurrence of this disease entity. Third, the plan IQ-based scoring method only calculates the target and OAR doses using CT density, but does not consider the delivery modality. In fact, no precise radiotherapy plan scoring system uses subjective criteria to evaluate each objective item. In other words, it would not be possible to define an absolute score for each treatment plan, although relative comparisons among different plans are possible. Our results should be interpreted in consideration of the aforementioned points, and they need to be verified through further clinical studies.

\section{Conclusions}

Our findings demonstrate that modern radiotherapy plans combining DIBH with VMAT or ${ }_{S} I M R T$ were significantly more beneficial than 3D plans for gastric MALToma by saving the OARs and enhancing conformity, regardless of concerns about increased MUs. Although whether DIBH, VMAT, or ${ }_{\text {SIMRT }}$ is more useful in the clinical setting is unclear, VMAT-FB seems to be better than 3D-DIBH regardless of target margins. Further studies are needed to confirm the relevance of the most practical plan considering clinical outcomes.

\section{Additional Files}

\begin{abstract}
Additional file 1: Figure S1. An example of the score template for the kidney and lung $D_{\text {mean }}(G y)$. Lower doses to the kidneys or lungs indicate better dosimetric distribution. Thus, a higher (superior) score can be acquired when the kidneys or lungs could be saved more, as the user set the template. (TIF $24 \mathrm{~kb}$ )

Additional file 2: Supplementary text $\mathbf{1}$. Significant results of statistical analyses using the Wilcoxon signed-rank test (D95). Supplementary text 2. Significant results of statistical analyses using the Wilcoxon signed-rank test (TV95). Supplementary text $\mathbf{3}$. Significant results of statistical analyses using the Wilcoxon signed-rank test (HI). Supplementary text 4. Significant results of statistical analyses using the Wilcoxon signed-rank test $(\mathrm{Cl})$. Supplementary text $\mathbf{5}$. Significant results of statistical analyses using the Wilcoxon signed-rank test ( $D_{\max }$ of the spinal cord).

Supplementary text 6 . Significant results of statistical analyses using the Wilcoxon signed-rank test ( $D_{\max }$ of the small bowel) (DOCX $\left.16 \mathrm{~kb}\right)$
\end{abstract}

\begin{abstract}
Abbreviations
3D-CRT: 3-dimensional conformal radiotherapy; AP/PA: Anterior-posterior/ posterior-anterior fields; CT: Computed tomography; CTV: Clinical target volume; DIBH: Deep-inspiration breath hold; FB: Free-breathing; GTV: Gross tumor volume; IMRT: Intensity-modulated radiotherapy; ITV: Internal target volume; MALToma: Mucosa-associated lymphoid tissue lymphoma; MSKCC: Memorial Sloan-Kettering Cancer Center; MU: Monitor unit; NCCN: National Comprehensive Cancer Network; OAR: Organ-at-risk; PQM: Plan quality metric; PTV: Planning target volume; sIMRT: step-and-shoot IMRT; VMAT: Volumetric-modulated arc therapy
\end{abstract}

\section{Acknowledgements}

This study was presented at the 37th Annual Meeting of the European SocieTy for Radiotherapy \& Oncology (ESTRO), Barcelona, Spain, April 2018. 


\section{Funding}

This work was supported by Ministry of Science, Korea through the R\&D program of NRF-2017M3A9E8029717.

\section{Availability of data and materials}

The datasets used and/or analyzed during the current study are available from the corresponding author on reasonable request.

\section{Authors' contributions}

SHC, SHP, JSK, and HIY conceived this study. SHC, SHP, JJBL, JGB, and HIY reviewed and checked the data of radiotherapy and dosimetric outcome. SHC, SHP, JSK, and HIY collected and organized the data. SHC and SHP analyzed the data and wrote the paper. All authors reviewed the manuscript. All authors read and approved the final manuscript.

\section{Ethics approval and consent to participate}

This study was approved by Institutional review board (IRB) of Yonsei University Health System (4-2017-1035). The patient records/information was anonymized and de-identified prior to analysis, and informed consent was not obtained from each participants.

\section{Consent for publication}

Not applicable.

\section{Competing interests}

The authors declare that they have no competing interests.

\section{Publisher's Note}

Springer Nature remains neutral with regard to jurisdictional claims in published maps and institutional affiliations.

\section{Author details}

${ }^{1}$ Department of Radiation Oncology, Yonsei Cancer Center, Yonsei University College of Medicine, 50-1 Yonsei-ro, Seodaemun-gu, Seoul 03722, South Korea. ${ }^{2}$ Department of Radiation Oncology, Jeju National University Hospital, Jeju University College of Medicine, Jeju, South Korea.

\section{Received: 30 April 2018 Accepted: 27 March 2019}

\section{Published online: 08 April 2019}

\section{References}

1. Park HJ, Park EH, Jung KW, et al. Statistics of hematologic malignancies in Korea: incidence, prevalence and survival rates from 1999 to 2008. Korean J Hematol. 2012;47(1):28-38.

2. Hahn JS, Kim YS, Lee YC, et al. Eleven-year experience of low grade lymphoma in Korea (based on REAL classification). Yonsei Med J. 2003;44(5): 757-70.

3. Schechter NR, Portlock CS, Yahalom J. Treatment of mucosa-associated Iymphoid tissue lymphoma of the stomach with radiation alone. J Clin Oncol. 1998;16(5):1916-21.

4. Tsang RW, Gospodarowicz MK, Pintilie M, et al. Localized mucosa-associated lymphoid tissue lymphoma treated with radiation therapy has excellent clinical outcome. J Clin Oncol. 2003;21(22):4157-64.

5. Vrieling $\mathrm{C}$, de Jong $\mathrm{D}$, Boot $\mathrm{H}$, et al. Long-term results of stomachconserving therapy in gastric MALT lymphoma. Radiother Oncol. 2008; 87(3):405-11.

6. Goda JS, Gospodarowicz M, Pintilie M, et al. Long-term outcome in localized extranodal mucosa-associated lymphoid tissue lymphomas treated with radiotherapy. Cancer. 2010;116(16):3815-24.

7. Wirth A, Gospodarowicz M, Aleman BM, et al. Long-term outcome for gastric marginal zone lymphoma treated with radiotherapy: a retrospective, multi-Centre, international Extranodal lymphoma study group study. Ann Oncol. 2013;24(5):1344-51.

8. Gobbi PG, Corbella F, Valentino F, et al. Complete long-term response to radiotherapy of gastric early-stage marginal zone lymphoma resistant to both anti-helicobacter pylori antibiotics and chemotherapy. Ann Oncol. 2009;20(3):465-8.

9. Horwitz SM, Zelenetz AD, Gordon LI, et al. NCCN guidelines insights: nonHodgkin's lymphomas, version 3.2016. J Natl Compr Cancer Netw. 2016; 14(9):1067-79.
10. Della Biancia C, Hunt M, Furhang E, et al. Radiation treatment planning techniques for lymphoma of the stomach. Int J Radiat Oncol Biol Phys. 2005;62(3):745-51.

11. Inaba K, Okamoto $H$, Wakita $A$, et al. Radiotherapy for gastric lymphoma: a planning study of 3D conformal radiotherapy, the halfbeam method, and intensity-modulated radiotherapy. J Radiat Res. 2014;55(6):1141-5.

12. Lim HW, Kim TH, Choi IJ, et al. Radiation therapy for gastric mucosaassociated lymphoid tissue lymphoma: dose-volumetric analysis and its clinical implications. Radiat Oncol J. 2016;34(3):193-201.

13. Bae SH, Kim DW, Kim MS, et al. Radiotherapy for gastric mucosaassociated lymphoid tissue lymphoma: dosimetric comparison and risk assessment of solid secondary cancer. Radiat Oncol J. 2017:35(1):78-89.

14. Nour AA, Alaradi A, Mohamed A, et al. Intensity modulated radiotherapy of upper abdominal malignancies: dosimetric comparison with 3D conformal radiotherapy and acute toxicity. Radiat Oncol. 2013;8:207.

15. Chung $\mathrm{JH}, \mathrm{Na} \mathrm{K}, \mathrm{Kim} \mid \mathrm{H}$. Benefit of volumetric-modulated arc therapy over three-dimensional conformal radiotherapy for stage I-II extranodal marginal zone B-cell lymphoma of mucosa-associated lymphoid tissue in the stomach: a dosimetric comparison. Radiat Oncol J. 2018;36(4): 332-40.

16. Ruben JD, Lancaster CM, Jones P, et al. A comparison of out-of-field dose and its constituent components for intensity-modulated radiation therapy versus conformal radiation therapy: implications for carcinogenesis. Int J Radiat Oncol Biol Phys. 2011:81(5):1458-64.

17. Matoba M, Oota K, Toyoda I, et al. Usefulness of 4D-CT for radiation treatment planning of gastric MZBCL/MALT. J Radiat Res. 2012;53(2):333-7.

18. Lee HY, Chang JS, Lee IJ, et al. The deep inspiration breath hold technique using Abches reduces cardiac dose in patients undergoing left-sided breast irradiation. Radiat Oncol J. 2013;31(4):239-46.

19. Gong $Y$, Wang $S$, Zhou $L$, et al. Dosimetric comparison using different multileaf collimeters in intensity-modulated radiotherapy for upper thoracic esophageal cancer. Radiat Oncol. 2010;5:65.

20. Feuvret L, Noel G, Mazeron JJ, et al. Conformity index: a review. Int J Radiat Oncol Biol Phys. 2006;64(2):333-42.

21. Clarke S, Goodworth J, Westhuyzen J, et al. Software-based evaluation of a class solution for prostate IMRT planning. Rep Pract Oncol Radiother. 2017; 22(6):441-9.

22. Fried DV, Chera BS, Das SK. Assessment of PlanlQ feasibility DVH for head and neck treatment planning. J Appl Clin Med Phys. 2017;18(5):245-50.

23. Liu $X$, Fang $H$, Tian $Y$, et al. Intensity modulated radiation therapy for earlystage primary gastric diffuse large B-cell lymphoma: Dosimetric analysis, clinical outcome, and quality of life. Int J Radiat Oncol Biol Phys. 2016;95(2): 712-20.

24. Yahalom J, Illidge $T$, Specht $L$, et al. Modern radiation therapy for extranodal lymphomas: field and dose guidelines from the international lymphoma radiation oncology group. Int J Radiat Oncol Biol Phys. 2015:92(1):11-31.

25. Reinartz G, Haverkamp U, Wullenkord R, et al. 4D-Listmode-PET-CT and 4DCT for optimizing PTV margins in gastric lymphoma : determination of intraand interfractional gastric motion. Strahlenther Onkol. 2016;192(5):322-32.

26. Nissen HD, Appelt AL. Improved heart, lung and target dose with deep inspiration breath hold in a large clinical series of breast cancer patients. Radiother Oncol. 2013:106(1):28-32.

27. Borst GR, Sonke JJ, den Hollander S, et al. Clinical results of image-guided deep inspiration breath hold breast irradiation. Int J Radiat Oncol Biol Phys. 2010;78(5):1345-51.

28. Remouchamps VM, Letts N, Vicini FA, et al. Initial clinical experience with moderate deep-inspiration breath hold using an active breathing control device in the treatment of patients with left-sided breast cancer using external beam radiation therapy. Int J Radiat Oncol Biol Phys. 2003;56(3): 704-15.

29. Lawler G, Leech M. Dose sparing potential of deep inspiration breath-hold technique for left breast Cancer radiotherapy organs-at-risk. Anticancer Res. 2017:37(2):883-90.

30. Aznar MC, Maraldo MV, Schut DA, et al. Minimizing late effects for patients with mediastinal Hodgkin lymphoma: deep inspiration breath-hold, IMRT, or both? Int J Radiat Oncol Biol Phys. 2015;92(1):169-74

31. Paumier A, Ghalibafian M, Gilmore J, et al. Dosimetric benefits of intensitymodulated radiotherapy combined with the deep-inspiration breath-hold technique in patients with mediastinal Hodgkin's lymphoma. Int J Radiat Oncol Biol Phys. 2012;82(4):1522-7. 
32. Paumier A, Ghalibafian M, Beaudre A, et al. Involved-node radiotherapy and modern radiation treatment techniques in patients with Hodgkin lymphoma. Int J Radiat Oncol Biol Phys. 2011;80(1):199-205.

33. Petersen PM, Aznar MC, Berthelsen AK, et al. Prospective phase II trial of image-guided radiotherapy in Hodgkin lymphoma: benefit of deep inspiration breath-hold. Acta Oncol. 2015;54(1):60-6.

34. Wang $H$, Milgrom SA, Dabaja BS, et al. Daily $C T$ guidance improves target coverage during definitive radiation therapy for gastric MALT lymphoma. Pract Radiat Oncol. 2017;7(6):e471-e8.

35. Goodman KA, Toner S, Hunt M, et al. Intensity-modulated radiotherapy for lymphoma involving the mediastinum. Int J Radiat Oncol Biol Phys. 2005; 62(1):198-206.

36. Parikh RR, Grossbard ML, Harrison LB, et al. Association of intensitymodulated radiation therapy on overall survival for patients with Hodgkin lymphoma. Radiother Oncol. 2016:118(1):52-9.

37. Voong KR, MCSpadden K, Pinnix CC, et al. Dosimetric advantages of a "butterfly" technique for intensity-modulated radiation therapy for young female patients with mediastinal Hodgkin's lymphoma. Radiat Oncol. 2014;9:94.

38. Illidge T, Specht L, Yahalom J, et al. Modern radiation therapy for nodal non-Hodgkin lymphoma-target definition and dose guidelines from the international lymphoma radiation oncology group. Int J Radiat Oncol Biol Phys. 2014:89(1):49-58

Ready to submit your research? Choose BMC and benefit from:

- fast, convenient online submission

- thorough peer review by experienced researchers in your field

- rapid publication on acceptance

- support for research data, including large and complex data types

- gold Open Access which fosters wider collaboration and increased citations

- maximum visibility for your research: over $100 \mathrm{M}$ website views per year

At BMC, research is always in progress.

Learn more biomedcentral.com/submissions 